\title{
Autologous CD5-specific CAR-28 zeta CAR T-cells
}

National Cancer Institute

\section{Source}

National Cancer Institute. Autologous CD5-specific CAR-28 zeta CART-cells. NCI

Thesaurus. Code C148490.

Autologous T-lymphocytes transduced with a retroviral vector expressing a chimeric antigen receptor (CAR) consisting of an anti-CD5 single chain variable fragment (scFv) coupled to the costimulatory signaling domain CD28 and the zeta chain of the T-cell receptor (TCR), with potential immunomodulating and antineoplastic activities. Upon transfusion, the autologous CD5-specific CAR-28 zeta CAR T-cells are directed to and induce selective toxicity in CD5-expressing tumor cells. The tumor-associated antigen (TAA) CD5 is a T-cell surface glycoprotein expressed on the surface of normal T-cells, and is overexpressed on various B- and T-cell malignancies; its expression is associated with poor prognosis. 